\title{
One-Pot Synthesis of Cyclic Carbonates from Aldehydes, Sulfur Ylide, and $\mathrm{CO}_{2}$
}

\author{
Ravindra D. Aher, B. Senthil Kumar, Arumugam Sudalai* \\ Chemical Engineering and Process Development Division, CSIR-National Chemical Laboratory, Pashan Road, Pune - 411 008, India \\ Fax +91(20)25902676; E-mail: a.sudalai@ncl.res.in
}

Received: 19.07.2013; Accepted after revision: 01.10.2013

\begin{abstract}
Treatment of aldehydes with sulfur ylide $\left(\mathrm{CH}_{2}=\mathrm{SOMe}_{2}\right.$ or $\left.\mathrm{CH}_{2}=\mathrm{SMe}_{2}\right)$, in the presence of $\mathrm{CO}_{2}(1 \mathrm{~atm})$ bubbled sequentially under mild conditions, produces cyclic carbonates in preparative yields. Sodium iodide formed in situ promotes the reaction between epoxide as intermediate and $\mathrm{CO}_{2}$ at ambient conditions, thus constituting a powerful metal-free synthesis of organic cyclic carbonates directly from aldehydes.
\end{abstract}

Key words: cyclization, epoxides, insertion, carbon dioxide, cyclic carbonates

Carbon dioxide is one of the major constituents of greenhouse gases, and to control its rapidly increased concentration in atmosphere is a major challenge for scientific communities. ${ }^{1}$ Global-warming concern has dramatically increased interest in using $\mathrm{CO}_{2}$ as a feedstock for the preparation of value-added chemicals. ${ }^{1 \mathrm{j}}$ Although $\mathrm{CO}_{2}$ is an attractive nonhazardous $\mathrm{C}_{1}$ synthon in abundance, its utilization in chemical transformations remains challenging because of its high thermodynamic stability and low chemical reactivity. ${ }^{2}$ In particular, the synthesis of lowenergy target molecules (e.g., organic carbonates) represents a promising alternative to overcome this thermodynamics. Organic cyclic carbonates are biodegradable chemicals that find tremendous applications as electrolytic materials in lithium ion batteries, polar aprotic solvents, intermediates in the production of pharmaceuticals and fine chemicals, as well as for developing engineered polymeric materials. ${ }^{3}$ Biologically active molecules that contain a cyclic carbonate moiety have also been isolated from various natural sources. ${ }^{4}$ In literature, cyclic carbonates are generally prepared by the cycloaddition of $\mathrm{CO}_{2}$ with epoxides in the presence of many activating reagents such as quaternary ammonium salts, ${ }^{5 a-c}$ azaphosphatranes, ${ }^{5 \mathrm{~d}}$ metal-salen complexes derived from $\mathrm{Al}, \mathrm{Zn}, \mathrm{Mg}$, and other first-row transition metals. ${ }^{6}$ Other methods of their synthesis include the reaction of $\mathrm{CO}_{2}$ with styrenes, ${ }^{7}$ propargyl alcohols, ${ }^{8}$ allyl alcohols, ${ }^{9}$ diols,${ }^{10}$ or halohydrins. ${ }^{11}$ Although these reported methods provide a simple route for the environmentally benign chemical fixation of $\mathrm{CO}_{2}$ to produce cyclic carbonates, these have certain limitations such as dependence on epoxides as starting materials, use of toxic or costly reagents, relatively high pressure and temperature, often requiring a tedious work-

SYNLETT 2014, 25, 0097-0101

Advanced online publication: 07.11.2013

DOI: 10.1055/s-0033-1340072; Art ID: ST-2013-B0676-L

C Georg Thieme Verlag Stuttgart · New York up procedure for their isolation. Sulfur ylides have been widely employed in the conversion of aldehydes into epoxides (Corey-Chaykovsky reaction) in organic synthesis, thereby serving as $\mathrm{C}_{1}$ carbon source under ambient conditions. ${ }^{12}$ In this note, we wish to disclose an efficient and metal-free, one-pot procedure for the synthesis of organic cyclic carbonates by the sequential reaction of aldehydes with sulfur ylides $\left(\mathrm{CH}_{2}=\mathrm{SOMe}_{2}\right.$ or $\left.\mathrm{CH}_{2}=\mathrm{SMe}_{2}\right)$ followed by its reaction with $\mathrm{CO}_{2}(1 \mathrm{~atm})$ in the absence of heavy-metal additives under ambient conditions (Scheme 1).

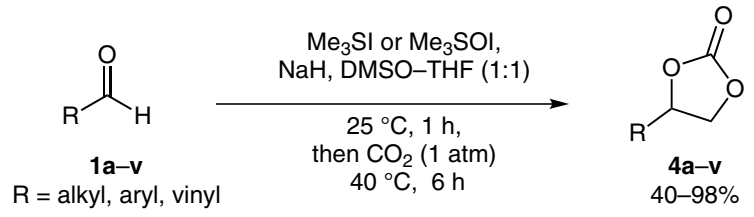

Scheme 1 Synthesis of cyclic carbonates from aldehydes, sulfur ylide, and $\mathrm{CO}_{2}$

Preliminary experiments have shown that when all the three components (aldehyde, sulfur ylide, and $\mathrm{CO}_{2}$ ) were treated together at $25^{\circ} \mathrm{C}$ in DMSO, a stable, colorless adduct $^{12}$ (mp 135-137 ${ }^{\circ} \mathrm{C}$ ) was obtained instantaneously due to the preferential reaction of sulfur ylide with $\mathrm{CO}_{2}$. It was then reasoned that $\mathrm{CO}_{2}$ could be bubbled sequentially after the in situ formation of epoxide from aldehyde and sulfur ylide.

The $\mathrm{CO}_{2}$ insertion into epoxide can be further facilitated by $\mathrm{NaI}$ as promoter formed in the reaction. However, when $\mathrm{CO}_{2}(1 \mathrm{~atm})$ was bubbled into the reaction mixture at $25^{\circ} \mathrm{C}$ after one hour (by which time the complete formation of epoxide has taken place), only epoxide $\mathbf{2 a}$ was isolated in $95 \%$ yield (Table 1, entry 1). At $40{ }^{\circ} \mathrm{C}$, it gave the desired cyclic carbonate $\mathbf{4 a}(30 \%)$ along with significant amount of iodo alcohol 3a (65\%). Subsequently it was found that the yield of $\mathbf{4 a}$ was, however, dependent linearly on temperature (Table 1, entries 3-5). At this stage, it was thought that by reducing the polarity of the solvent, the nucleophilicity of the iodo alkoxide 3 (Scheme 2) could be increased for its reaction with $\mathrm{CO}_{2}$ so as to maximize the yield of $\mathbf{4 a} .{ }^{11}$ Thus, when the reaction was carried out with a relatively low polar solvent system (DMSO-THF, $1: 1)$ at $25^{\circ} \mathrm{C}$ under $\mathrm{CO}_{2}(1 \mathrm{~atm})$, no cyclic carbonate was observed; only epoxide $\mathbf{2 a}$ was again obtained in $96 \%$ yield. Finally, at $40{ }^{\circ} \mathrm{C}$, a dramatic improvement in yield of $4 \mathrm{a}(94 \%)$ was achieved with the 
Table 1 Optimization Parameters for Cyclic Carbonates ${ }^{\mathrm{a}}$

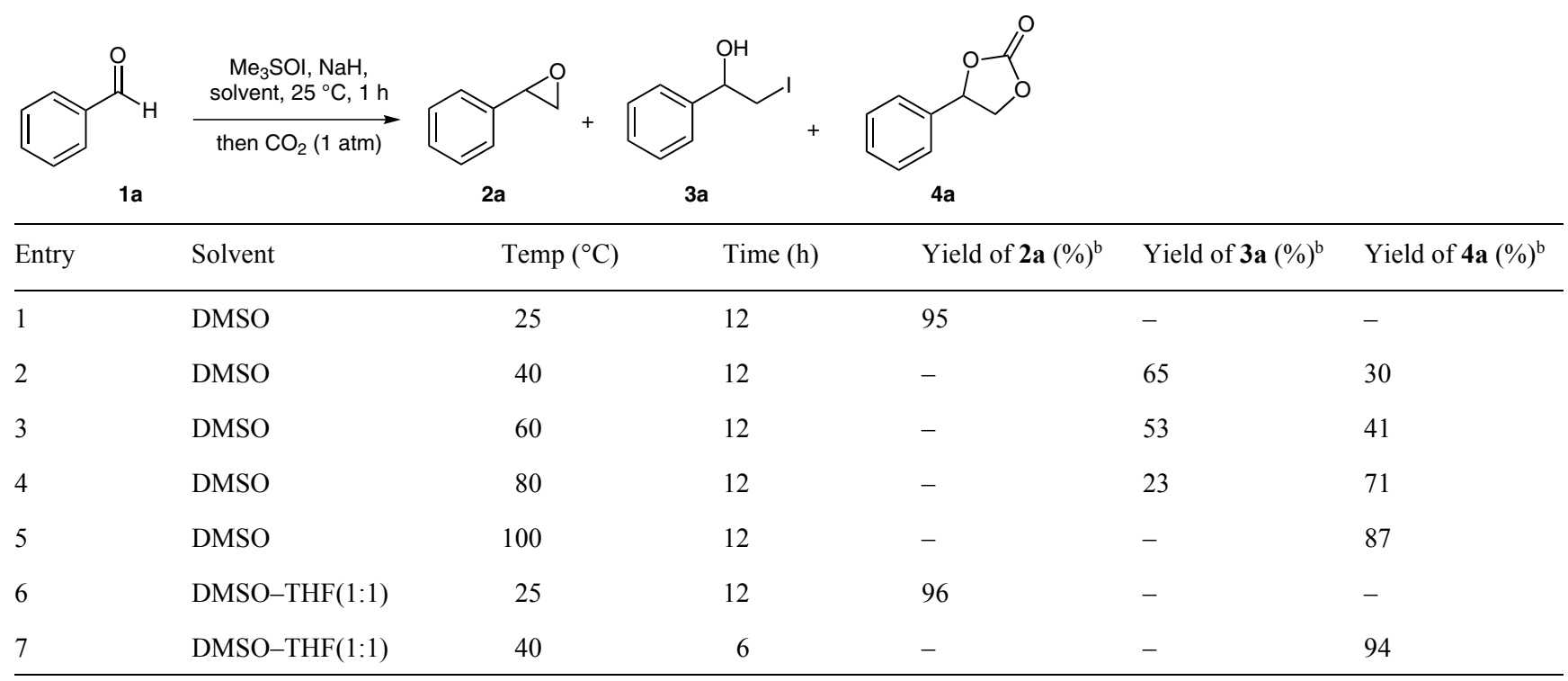

a Aldehyde ( $3 \mathrm{mmol}), \mathrm{NaH}(3.3 \mathrm{mmol}), \mathrm{Me}_{3} \mathrm{SOI}(3.3 \mathrm{mmol})$, solvent $(20 \mathrm{~mL})$.

${ }^{\mathrm{b}}$ Isolated yield after column chromatographic purification.

same solvent system Table 1, entry 7). Unfortunately, other bases $(\mathrm{KOH}, \mathrm{NaOH}, \mathrm{KO} t-\mathrm{Bu})^{13}$ were not as effective as $\mathrm{NaH}$. With the optimized reaction conditions in hand, ${ }^{16}$ we then examined the substrate scope of the reaction (Table 2). Even lower members of cyclic carbonates of commercial importance (4b and $\mathbf{4 c}$ ) can be prepared in moderate to good yields. Further, aldehydes bearing halo, cyano, nitro, methoxy, and methylene dioxy groups on the aromatic nucleus and functionalized aliphatic aldehydes underwent this sequential reaction smoothly to afford the corresponding cyclic carbonates $\mathbf{4 a}-\mathbf{v}$ in high yields. Among the substrates screened, aldehdyes with halo $\mathbf{4 k}-$ $\mathbf{n}$ and cyano $\mathbf{4 t}$ substituents on the aromatic nucleus were found to be the best substrates for this methodology.

Volatile aliphatic and conjugated aldehydes ${ }^{14}$ were also converted into the corresponding cyclic carbonates $\mathbf{4 b}-\mathbf{h}$ in high yields (Table 2, entries 2-8). Also, chiral amino aldehyde $1 \mathbf{u}$ gave the corresponding cyclic carbonate $4 \mathbf{u}$ efficiently under the reaction conditions (Table 2, entry 21). Cyclic carbonates $\mathbf{4 a}-\mathbf{v}$ have exhibited a strong IR absorption in the range $1792-1822 \mathrm{~cm}^{-1}$ due to the $\mathrm{C}=\mathrm{O}$ stretching vibration of the cyclic carbonate moiety.

Table 2 Cyclic Carbonates from Aldehydes and $\mathrm{CH}_{2}=\mathrm{SOMe}_{2}$ or $\mathrm{CH}_{2}=\mathrm{SMe}_{2}$ with $\mathrm{CO}_{2}{ }^{\mathrm{a}}$

\begin{tabular}{lll}
\hline Entry & Aldehydes 1 & $\begin{array}{l}\text { Yield of cyclic } \\
\text { carbonates }(\%)^{\mathbf{b}}\end{array}$ \\
\hline 1 & benzaldehyde (1a) & $\mathbf{4 a} 94$ \\
2 & acetaldehyde (1b) & $\mathbf{4 b} 40$ \\
3 & propionaldehyde (1c) & $\mathbf{4 c} 65$ \\
4 & isobutyraldehyde (1d) & $\mathbf{4 d ~} 73$ \\
5 & $n$-pentanal (1e) & $\mathbf{4 e} 89$
\end{tabular}

Table 2 Cyclic Carbonates from Aldehydes and $\mathrm{CH}_{2}=\mathrm{SOMe}_{2}$ or $\mathrm{CH}_{2}=\mathrm{SMe}_{2}$ with $\mathrm{CO}_{2}{ }^{\mathrm{a}}$ (continued)

\begin{tabular}{|c|c|c|}
\hline Entry & Aldehydes 1 & $\begin{array}{l}\text { Yield of cyclic } \\
\text { carbonates }(\%)^{b}\end{array}$ \\
\hline 6 & $n$-heptanal (1f) & 4f 94 \\
\hline $7^{\mathrm{b}}$ & acrolein $(\mathbf{1 g})$ & $4 g 65$ \\
\hline $8^{\mathrm{b}}$ & cinnamaldehyde (1h) & 4h 98 \\
\hline 9 & 2-methoxybenzaldehyde (1i) & $4 \mathbf{i} 85$ \\
\hline 10 & 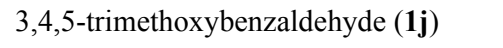 & $\mathbf{4 j} 90$ \\
\hline 11 & 4-fluorobenzaldehyde (1k) & $4 \mathbf{k} 90$ \\
\hline 12 & 4-chlorobenzaldehyde (11) & 4187 \\
\hline 13 & 2-bromobenzaldehyde (1m) & $4 \mathbf{m} 96$ \\
\hline 14 & 4-trifluoromethylbenzaldehyde (1n) & 4n 98 \\
\hline 15 & 2-nitrobenzaldehyde (1o) & 4079 \\
\hline 16 & 3-nitrobenzaldehyde (1p) & $4 p 75$ \\
\hline 17 & piperonal (1q) & 4q 86 \\
\hline 18 & 3-benzyloxy-1-propanal (1r) & $4 \mathbf{r} 80$ \\
\hline 19 & 3-phenylpropanal (1s) & 4s 77 \\
\hline 20 & $3,4-(\mathrm{OMe})_{2}-2-\mathrm{CN}-$ phenyl-propanal $(\mathbf{1 t})$ & 4t 83 \\
\hline 21 & $(S)$-( $\alpha$-NHBoc)-3-phenyl-propanal (1u) & $4 \mathbf{u} 79$ \\
\hline 22 & 4-thiomethylbenzaldehyde (1v) & $4 v 90$ \\
\hline
\end{tabular}

${ }^{a}$ Reaction conditions: aldehyde ( $\left.3 \mathrm{mmol}\right), \mathrm{NaH}$ ( $\left.3.3 \mathrm{mmol}\right), \mathrm{Me}_{3} \mathrm{SOI}$ (3.3 mmol), DMSO $(10 \mathrm{~mL}), \mathrm{THF}(10 \mathrm{~mL}), 25^{\circ} \mathrm{C}, 1 \mathrm{~h}$, then $\mathrm{CO}_{2}$ bubbling $40^{\circ} \mathrm{C}, 6 \mathrm{~h}$.

${ }^{b}$ Dimethylsulfonium methylide was used instead of dimethyloxosulfonium methylide. 
For comparative studies, it was of interest to investigate the effect of temperature on cycloaddition of styrene epoxide with $\mathrm{CO}_{2}$ using various alkali metal iodides. Thus, when styrene epoxide was treated with $\mathrm{CO}_{2}$ (1.0133 bar) in the presence of $\mathrm{NaI}(100 \mathrm{~mol} \%)$ in DMSO-THF (1:1) at $25^{\circ} \mathrm{C}$ for 12 hours, no reaction took place. However, when the same reaction was carried out at $40{ }^{\circ} \mathrm{C}$ for six hours, the corresponding styrene cyclic carbonate was obtained in $95 \%$ yield. Also, other alkali metal iodides (KI and LiI, in stoichiometric amount) under the same reaction conditions, could be employed to give the styrene cyclic carbonate in $73 \%$ and $83 \%$ yield, respectively. NaI could also be used in catalytic amount $(10 \mathrm{~mol} \%)$ to give styrene cyclic carbonate in $91 \%$ yield, although it took 38 hours to completion. From the above investigations, we strongly believe that a slightly higher temperature $\left(40^{\circ} \mathrm{C}\right)$ is needed for the activation of styrene epoxide by alkali metal iodide.

Based on the above observations, a probable mechanistic pathway for the formation of cyclic carbonates is shown in Scheme 2. ${ }^{15}$ Firstly, aldehydes $\mathbf{1 a}-\mathbf{v}$ react with sulfur ylide (generated in situ from $\mathrm{O}=\mathrm{SMe}_{3} \mathrm{I}$ and $\mathrm{NaH}$ in DMSO) to form epoxide 2 which was isolated and characterized. Subsequently, $\mathrm{NaI}$ formed in situ in the reaction medium promotes the regioselective ring opening of epoxide 2 to give iodo alkoxide complex 3 (confirmed by isolation and characterization of iodoalcohol $\mathbf{3 a}$, see Table 1). This is followed by its simultaneous insertion with $\mathrm{CO}_{2}$ producing the intermediate $\mathbf{I}$, cyclization of which results in the formation of cyclic carbonates $4 \mathbf{a}-\mathbf{v}$.
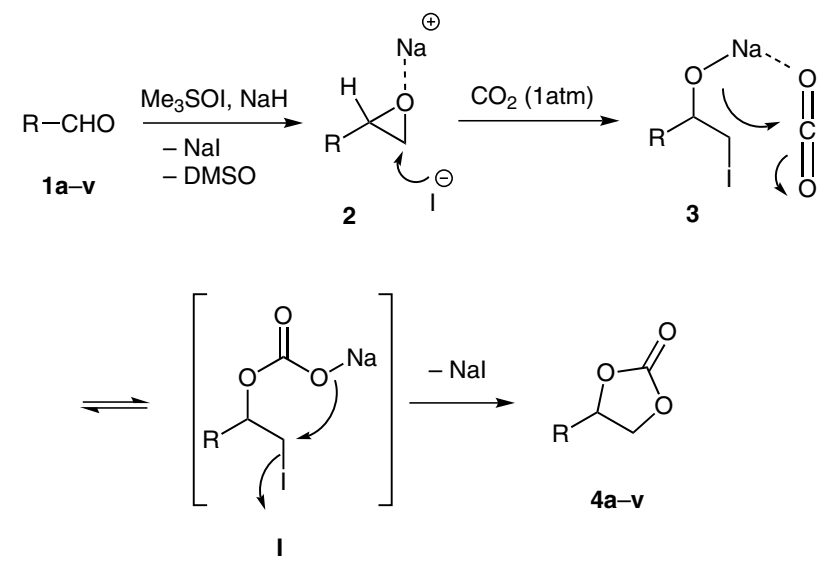

Scheme 2 Pathway for the formation of cyclic carbonates from aldehydes, sulfur ylide, and $\mathrm{CO}_{2}$

In summary, we have described, for the first time, a novel 'one-pot' procedure that involves reaction of aldehydes $\mathbf{1 a}-\mathbf{v}$ with sulfur ylides, followed by $\mathrm{CO}_{2}$ insertion in a sequential fashion leading to the high-yield synthesis of cyclic carbonates $\mathbf{4 a}-\mathbf{v}$. The salient features of the methodology are as follows: (1) unprecedented one-pot synthesis of organic cyclic carbonates directly from aldehydes; (2) inexpensive and commercially available starting materials; (3) metal-free synthesis; (4) water-soluble NaI acts as a promoter; (5) milder reaction conditions; (6) functional-group tolerance; and (7) high yields of cyclic carbonates.

\section{Acknowledgment}

We sincerely thank CSIR, New Delhi, India (NWP-0021B) for financial support. RDA and BSK thank CSIR, New Delhi, India for research fellowships. Authors also thank Dr V. V. Ranade, Chair, Chemical Eng. \& Process Develop. Div. and Dr B. D. Kulkarni, Distinguished Scientist for their constant encouragement and support.

Supporting Information for this article is available online at http://www.thieme-connect.com/ejournals/toc/synlett.

\section{References and Notes}

(1) (a) Sakakura, T.; Choi, J. C.; Yasuda, H. Chem. Rev. 2007, 107, 2365. (b) Braunstein, P.; Matt, D.; Nobel, D. Chem. Rev. 1988, 88, 747. (c) Behr, A. Angew. Chem., Int. Ed. Engl. 1988, 27, 661. (d) Jessop, P. G.; Joo, F.; Tai, C. C. Coord. Chem. Rev. 2004, 248, 2425. (e) Darensbourg, D. J. Chem. Rev. 2007, 107, 2388. (f) Eghbali, N.; Li, C. J. Green Chem. 2007, 9, 213. (g) Riduan, S. N.; Zhang, Y. Dalton Trans. 2010, 39, 3347. (h) Sakakura, T.; Kohon, K. Chem. Commun. 2009, 1312. (i) Aher, R. D. Gade M. H. Reddy R. S.; Sudalai, A. Indian J. Chem., Sect. A: Inorg., Bio-inorg., Phys., Theor. Anal. Chem. 2012, 51, 1325. (j) Olah, G. A. Angew. Chem. Int. Ed. 2005, 44, 2636.

(2) (a) Zevenhoven, R.; Eloneva, S.; Teir, S. Catal. Today 2006, 115, 73. (b) Aresta, M.; Dibenedetto, A.; Tommasi, I. Energy Fuels 2001, 15, 269. (c) Tundo, P.; Selva, M. Acc. Chem. Res. 2002, 35, 706. (d) Aida, T.; Inoue, S. Acc. Chem. Res. 1998, 29, 39. (e) Riduan, S. N.; Zhang, Y.; Ying, J. Y. Angew. Chem. Int. Ed. 2009, 48, 3322. (f) Gu, L.; Zhang, Y. J. Am. Chem. Soc. 2010, 132, 914.

(3) (a) Schaffner, B.; Schaffner, F.; Verevkin, S. P.; Borner, A. Chem. Rev. 2010, 110, 4554. (b) Shaikh, A. A. G.; Sivaram, S. Chem. Rev. 1996, 96, 951. (c) Clements, J. H. Ind. Eng. Chem. Res. 2003, 42, 663.

(4) (a) Davis, R. A.; Andjic, V.; Kotiw, M.; Shivas, R. G. Phytochemistry 2005, 66, 2771. (b) Liu, Z.; Jensen, P. R.; Fenical, W. Phytochemistry 2003, 64, 571.

(5) (a) Ream, B. C. US 4,877,886, 1989. (b) McMullen, C. H.; Nelson, J. R.; Ream, B. C.; Sims, J. A. J. US 4,314,945, 1982. (c) Peppel, W. J. J. Ind. Eng. Chem. 1958, 50, 767. (d) Chatelet, B.; Joucla, L.; Dutasta, J.-P.; Martinez, A.; Szeto, K. C.; Dufaud, V. J. Am. Chem. Soc. 2013, 135, 5348.

(6) (a) Lu, X.-B.; He, R.; Bai, C.-X. J. Mol. Catal. A: Chem. 2002, 186, 1. (b) Lu, X.-B.; Liang, B.; Zhang, Y.-J.; Tian, Y.-Z.; Wang, Y.-M.; Bai, C.-X.; Wang, H.; Zhang, R. J. Am. Chem. Soc. 2004, 126, 3732. (c) Melendez, J.; North, M.; Pasquale, R. Eur. J. Inorg. Chem. 2007, 3323. (d) Melendez, J.; North, M.; Villuendas, P. Chem. Commun. 2009, 2577. (e) North, M.; Pasquale, R. Angew. Chem. Int. Ed. 2009, 48, 2946. (f) Paddock, R. L.; Nguyen, S. T. Chem. Commun. 2004, 1622. (g) Darensbourg, D. J.; Bottarelli, P.; Andreatta, J. R. Macromolecules 2007, 40, 7727. (h) Darensbourg, D. J.; Fang, C. C.; Rodgers, J. L. Organometallics 2004, 23, 924. (i) Lu, X. B.; Pan, Y. Z.; Ji, D. F.; He, R. Chin. Chem. Lett. 2000, 11, 589.

(7) (a) Wang, J. L.; Wang, J. Q.; He, L. N.; Dou, X. Y.; Wu, F. Green Chem. 2008, 10, 1218. (b) Bai, D. S.; Jing, H. W. Green Chem. 2010, 12, 39. 
(8) Satoshi, K.; Shunsuke, Y.; Yuudai, S.; Wataru, Y.; Hau, M. C.; Kosuke, F.; Kohei, S.; Izumi, I.; Taketo, I.; Tohru, Y. Bull. Chem. Soc. Jpn. 2011, 84, 698.

(9) Minakata, S.; Sasaki, I.; Ide, T. Angew. Chem. Int. Ed. 2010 49, 1309.

(10) (a) Du, Y.; Kong, D. L.; Wang, H. Y.; Cai, F.; Tian, J. S.; Wang, J. Q.; He, L. N. J. Mol. Catal. A: Chem. 2005, 241, 233. (b) Du, Y.; He, L. N. Catal. Commun. 2008, 9, 1754.

(11) Peng, Y.; Xueqin, T.; Huanwang, J.; Shuhui, D.; Xiaoxuan, W.; Zhongli, L. J. Org. Chem. 2011, 76, 2459.

(12) (a) Corey, E. J.; Chaykovsky, M. J. Am. Chem. Soc. 1965, 87,1353 . (b) Melting point was found to be in agreement with the reported value.

(13) (a) Ylide generation failed with $\mathrm{NaOH}$. (b) Diol was obtained in $48 \%$ yield with $\mathrm{KOH}$. (c) Trace amount $(5 \%)$ of epoxide was obtained using $\mathrm{KO} t$-Bu as base.

(14) Dimethylsulfonium methylide was used instead of dimethyloxosulfonium methylide.

(15) North, M.; Pasquale, R. Angew. Chem. Int. Ed. 2009, 48, 2946.

(16) General Description

Solvents were purified and dried by standard procedures before use; $\mathrm{PE}$ of boiling range $60-80{ }^{\circ} \mathrm{C}$ was used. Melting points are uncorrected and recorded on a Buchi B-542 instrument. ${ }^{1} \mathrm{H}$ NMR and ${ }^{13} \mathrm{C}$ NMR spectra were recorded on Bruker AC-200 spectrometer unless mentioned otherwise. Elemental analysis was carried out on a Carlo Erba CHNS$\mathrm{O}$ analyzer. Infrared spectra were recorded on Shimadzu FTIR-8400 spectrometer and absorption is expressed in $\mathrm{cm}^{-1}$. HRMS data for new compounds were recorded on a Thermo Scientific Q-Exactive, Accela 1250 pump. Purification was done using column chromatography (230-400 mesh).

General Experimental Procedure for the Preparation of Cyclic Carbonates 4a-v

$\mathrm{NaH}(0.132 \mathrm{~g}, 3.3 \mathrm{mmol}$; previously washed with anhydrous $\mathrm{PE}$ to remove oil) was taken in an oven-dried three-necked flask, followed by addition of anhydrous DMSO $(10 \mathrm{~mL})$ through a septum to it, and the whole slurry was stirred at 25 ${ }^{\circ} \mathrm{C}$ under $\mathrm{N}_{2}$ atmosphere. Solid $\mathrm{Me}_{3} \mathrm{SOI}(0.726 \mathrm{~g}, 3.3 \mathrm{mmol})$ was added to the slurry with stirring over a period of $5 \mathrm{~min}$ via a solid addition funnel until it becomes a homogeneous solution. A solution of aldehyde $1 \mathbf{a}-\mathbf{v}(3 \mathrm{mmol})$, dissolved in anhydrous THF $(10 \mathrm{~mL})$, was added dropwise to the reaction mixture. After $1 \mathrm{~h}, \mathrm{CO}_{2}(1 \mathrm{~atm})$ was then bubbled slowly via a needle into the reaction mixture, (after ascertaining that aldehyde was completely converted into epoxide, monitored by TLC) at $40{ }^{\circ} \mathrm{C}$, for $6 \mathrm{~h}$. Water $(10 \mathrm{~mL})$ was added to quench the reaction. It was then extracted with EtOAc $(3 \times$ $20 \mathrm{~mL}$ ); the organic layer was washed with brine and dried over anhydrous $\mathrm{Na}_{2} \mathrm{SO}_{4}$ and the solvent concentrated, product purified by silica gel column chromatography (100200 mesh) using PE and EtOAc (70:30) as eluent to afford pure cyclic carbonates $\mathbf{4 a}-\mathbf{v}$.

\section{4-Phenyl-1,3-dioxolan-2-one (4a)}

Yield 94\% (463 mg, $2.82 \mathrm{mmol}$ ); colorless solid; mp 53$54{ }^{\circ} \mathrm{C}$ (lit. mp 51-53 $\left.{ }^{\circ} \mathrm{C}\right)$. IR $\left(\mathrm{CHCl}_{3}\right): v_{\max }=769,1068$, $1168,1328,1458,1812 \mathrm{~cm}^{-1}$. ${ }^{1} \mathrm{H}$ NMR $\left(200 \mathrm{MHz}, \mathrm{CDCl}_{3}\right)$ : $\delta=4.29(\mathrm{t}, J=7.9 \mathrm{~Hz}, 1 \mathrm{H}), 4.77(\mathrm{t}, J=7.9 \mathrm{~Hz}, 1 \mathrm{H}), 5.64$ $(\mathrm{t}, J=7.9 \mathrm{~Hz}, 1 \mathrm{H}), 7.32-7.42(\mathrm{~m}, 5 \mathrm{H}) .{ }^{13} \mathrm{C}$ NMR $(50 \mathrm{MHz}$, $\left.\mathrm{CDCl}_{3}\right): \delta=70.9,77.8,125.7,129.0,129.4,135.8,154.6$. Anal. Calcd (\%) for $\mathrm{C}_{9} \mathrm{H}_{8} \mathrm{O}_{3}: \mathrm{C}, 65.85 ; \mathrm{H}, 4.91$. Found: $\mathrm{C}$, $65.84 ; \mathrm{H}, 4.90$.

\section{4-Methyl-1,3-dioxolan-2-one (4b)}

Yield 40\% (122.5 mg, $1.2 \mathrm{mmol})$; colorless oil; IR $\left(\mathrm{CHCl}_{3}\right)$ : $v_{\max }=711,776,1051,1120,1183,1354,1389,1793 \mathrm{~cm}^{-1}$. ${ }^{1} \mathrm{H}$ NMR $\left(200 \mathrm{MHz}, \mathrm{CDCl}_{3}\right): \delta=1.49(\mathrm{~d}, J=6.1 \mathrm{~Hz}, 3 \mathrm{H})$, $4.02(\mathrm{dd}, J=8.2,1.0 \mathrm{~Hz}, 1 \mathrm{H}), 4.55(\mathrm{t}, J=8.0 \mathrm{~Hz}, 1 \mathrm{H})$,
4.77-4.94 (m, $\left.1 \mathrm{H}) .{ }^{13} \mathrm{C} \mathrm{NMR} \mathrm{(100} \mathrm{MHz,} \mathrm{CDCl}_{3}\right): \delta=19.0$, 70.4, 73.4, 154.8. Anal. Calcd (\%) for $\mathrm{C}_{4} \mathrm{H}_{6} \mathrm{O}_{3}: \mathrm{C}, 47.06 ; \mathrm{H}$, 5.92. Found: C, 47.04; H, 5.91

\section{4-Ethyl-1,3-dioxolan-2-one (4c)}

Yield 65\% (226.4 mg, $1.95 \mathrm{mmol}$ ); colorless oil. IR $\left(\mathrm{CHCl}_{3}\right): v_{\max }=1060,1177,1377,1797 \mathrm{~cm}^{-1} .{ }^{1} \mathrm{H}$ NMR $(200$ $\left.\mathrm{MHz}, \mathrm{CDCl}_{3}\right): \delta=1.03(\mathrm{t}, J=7.4 \mathrm{~Hz}, 3 \mathrm{H}), 1.72-1.87(\mathrm{~m}, 2$ $\mathrm{H}), 4.1(\mathrm{dd}, J=8.2,1.5 \mathrm{~Hz}, 1 \mathrm{H}), 4.55(\mathrm{t}, J=8.2 \mathrm{~Hz}, 1 \mathrm{H})$, 4.63-4.73 (m, $1 \mathrm{H}) .{ }^{13} \mathrm{C}$ NMR (50 MHz, $\left.\mathrm{CDCl}_{3}\right): \delta=7.9$, 26.2, 68.5, 77.6, 154.6. Anal. Calcd (\%) for $\mathrm{C}_{5} \mathrm{H}_{8} \mathrm{O}_{3}: \mathrm{C}$, 51.72; H, 6.94. Found: C, 51.75; H, 6.91.

\section{4-Isopropyl-1,3-dioxolan-2-one (4d)}

Yield $73 \%$ (285 mg, $2.19 \mathrm{mmol})$; colorless oil. IR $\left(\mathrm{CHCl}_{3}\right)$ : $v_{\max }=1075,1175,1392,1789 \mathrm{~cm}^{-1} .{ }^{1} \mathrm{H}$ NMR $(200 \mathrm{MHz}$, $\left.\mathrm{CDCl}_{3}\right): \delta=0.95(\mathrm{~d}, J=6.8 \mathrm{~Hz}, 3 \mathrm{H}), 1.04(\mathrm{~d}, J=6.5 \mathrm{~Hz}, 3$ H), $1.90-2.00(\mathrm{~m}, 1 \mathrm{H}), 4.12-4.18(\mathrm{~m}, 1 \mathrm{H}), 4.37-4.53(\mathrm{~m}, 2$ H). $\left.{ }^{13} \mathrm{C} \mathrm{NMR} \mathrm{(50} \mathrm{MHz,} \mathrm{CDCl}_{3}\right): \delta=16.4,16.8,31.4,67.2$, 80.9, 154.6. HRMS $\left(\mathrm{ESI}^{+}\right): \mathrm{m} / z=$ calcd for $\left(\mathrm{C}_{6} \mathrm{H}_{10} \mathrm{O}_{3}\right)^{+}[\mathrm{M}+$ $\mathrm{H}]^{+}$: 131.0709; found: 131.0708. Anal. Calcd (\%) for $\mathrm{C}_{6} \mathrm{H}_{10} \mathrm{O}_{3}$ : C, 55.37; $\mathrm{H}, 7.75$. Found: $\mathrm{C}, 55.40 ; \mathrm{H}, 7.71$. 4-Butyl-1,3-dioxolan-2-one (4e)

Yield $89 \%$ (384.9 mg, $2.6 \mathrm{mmol})$; gum. IR $\left(\mathrm{CHCl}_{3}\right): v_{\max }=$ 1066, 1173, $1797 \mathrm{~cm}^{-1} .{ }^{1} \mathrm{H}$ NMR $\left(200 \mathrm{MHz}, \mathrm{CDCl}_{3}\right): \delta=$ $0.88-0.95$ (m, $3 \mathrm{H}), 1.23-1.40(\mathrm{~m}, 3 \mathrm{H}), 1.68-1.80(\mathrm{~m}, 3 \mathrm{H})$, $4.05(\mathrm{dd}, J=8.2,1.0 \mathrm{~Hz}, 1 \mathrm{H}), 4.51(\mathrm{t}, J=8.2 \mathrm{~Hz}, 1 \mathrm{H})$, 4.62-4.76 (m, $1 \mathrm{H}) .{ }^{13} \mathrm{C}$ NMR $\left(50 \mathrm{MHz}, \mathrm{CDCl}_{3}\right): \delta=13.8$, 22.2, 26.4, 33.6, 69.3, 76.9, 154.9. Anal. Calcd (\%) for $\mathrm{C}_{7} \mathrm{H}_{12} \mathrm{O}_{3}$ : C, 58.32; H, 8.39. Found: $\mathrm{C}, 58.34 ; \mathrm{H}, 8.38 \%$.

\section{4-Hexyl-1,3-dioxolan-2-one (4f)}

Yield 94\% (486 mg, $2.82 \mathrm{mmol})$; gum. IR $\left(\mathrm{CHCl}_{3}\right): v_{\max }=$ $772,1065,1170,1802 \mathrm{~cm}^{-1} .{ }^{1} \mathrm{H}$ NMR $\left(200 \mathrm{MHz}, \mathrm{CDCl}_{3}\right): \delta$ $=0.86-0.93(\mathrm{~m}, 3 \mathrm{H}), 1.30-1.49(\mathrm{~m}, 8 \mathrm{H}), 1.69-1.80(\mathrm{~m}, 2$ $\mathrm{H}), 4.06(\mathrm{t}, J=7.2 \mathrm{~Hz}, 1 \mathrm{H}), 4.51(\mathrm{t}, J=8.0 \mathrm{~Hz}, 1 \mathrm{H}), 4.62$ $4.76(\mathrm{~m}, 1 \mathrm{H}) .{ }^{13} \mathrm{C} \mathrm{NMR}\left(50 \mathrm{MHz}, \mathrm{CDCl}_{3}\right): \delta=13.9,22.4$, 24.3, 28.7, 31.4, 33.8, 69.2, 76.8, 154.8. Anal. Calcd (\%) for $\mathrm{C}_{9} \mathrm{H}_{16} \mathrm{O}_{3}$ : C, 62.77; H, 9.36. Found: C, 62.73; H, 9.40.

4-Vinyl-1,3-dioxolan-2-one (4g)

Yield 65\% (222.5 mg, $1.95 \mathrm{mmol})$; yellow oil. IR $\left(\mathrm{CHCl}_{3}\right)$ : $v_{\max }=772,991,1060,1168,1385,1510,1805 \mathrm{~cm}^{-1} .{ }^{1} \mathrm{H}$ NMR $\left(200 \mathrm{MHz}, \mathrm{CDCl}_{3}\right): \delta=4.17(\mathrm{t}, J=7.8 \mathrm{~Hz}, 1 \mathrm{H}), 4.61$ $(\mathrm{t}, J=8.4 \mathrm{~Hz}, 1 \mathrm{H}), 5.12(\mathrm{dd}, J=14.9,7.5 \mathrm{~Hz}, 1 \mathrm{H}), 5.48$ (t, $J=16.5 \mathrm{~Hz}, 2 \mathrm{H}), 5.83-6.0(\mathrm{~m}, 1 \mathrm{H}) .{ }^{13} \mathrm{C}$ NMR $(50 \mathrm{MHz}$, $\left.\mathrm{CDCl}_{3}\right): \delta=68.8,76.8,120.9,132.2,154.2 . \mathrm{HRMS}\left(\mathrm{ESI}^{+}\right)$: $m / z$ calcd for $\left(\mathrm{C}_{5} \mathrm{H}_{6} \mathrm{O}_{3}\right)^{+}\left[\mathrm{M}+\mathrm{H}^{+}: 115.0396\right.$; found:

115.0392; Anal. Calcd (\%) for $\mathrm{C}_{5} \mathrm{H}_{6} \mathrm{O}_{3}: \mathrm{C}, 52.63 ; \mathrm{H}, 5.30$. Found: C, 52.61; H, 5.32.

\section{(E)-4-Styryl-1,3-dioxolan-2-one (4h)}

Yield 98\% (559 mg, $2.94 \mathrm{mmol}$ ); colorless solid; mp 115$116^{\circ} \mathrm{C}$. IR $\left(\mathrm{CHCl}_{3}\right): v_{\max }=1049,1070,1168,1648,1800$ $\mathrm{cm}^{-1} .{ }^{1} \mathrm{H}$ NMR $\left(200 \mathrm{MHz}, \mathrm{CDCl}_{3}\right): \delta=4.23(\mathrm{t}, J=8.0 \mathrm{~Hz}, 1$ H), $4.64(\mathrm{t}, J=8.2 \mathrm{~Hz}, 1 \mathrm{H}), 5.26(\mathrm{q}, J=7.9 \mathrm{~Hz}, 1 \mathrm{H}), 6.15$ $(\mathrm{dd}, J=15.7,7.7 \mathrm{~Hz}, 1 \mathrm{H}), 6.73(\mathrm{~d}, J=15.7 \mathrm{~Hz}, 1 \mathrm{H}), 7.26$ $7.40(\mathrm{~m}, 5 \mathrm{H}) .{ }^{13} \mathrm{C}$ NMR $\left(50 \mathrm{MHz}, \mathrm{CDCl}_{3}\right): \delta=69.2,77.6$, 122.4, 126.9, 128.8, 129.0, 134.8, 136.6, 154.4. HRMS $\left(\mathrm{ESI}^{+}\right): \mathrm{m} / z$ calcd for $\left(\mathrm{C}_{11} \mathrm{H}_{10} \mathrm{O}_{3}\right)^{+}[\mathrm{M}+\mathrm{Na}]^{+}: 213.0527$; found: 213.0522. Anal. Calcd (\%) for $\mathrm{C}_{11} \mathrm{H}_{10} \mathrm{O}_{3}: \mathrm{C}, 69.46 ; \mathrm{H}$, 5.30. Found: C, 69.41; H, 5.33 .

\section{4-(2-Methoxyphenyl)-1,3-dioxolan-2-one (4i)}

Yield $85 \%$ (495 mg, $2.55 \mathrm{mmol})$; gum. IR $\left(\mathrm{CHCl}_{3}\right): v_{\max }=$ $757,1076,1166,1249,1494,1812 \mathrm{~cm}^{-1}$. ${ }^{1} \mathrm{H}$ NMR $(200$ $\left.\mathrm{MHz}, \mathrm{CDCl}_{3}\right): \delta=3.86(\mathrm{~s}, 3 \mathrm{H}), 4.25(\mathrm{dd}, J=8.4,1.1 \mathrm{~Hz}, 1$ H), $4.82(\mathrm{t}, J=8.4 \mathrm{~Hz}, 1 \mathrm{H}), 5.81(\mathrm{t}, J=8.0 \mathrm{~Hz}, 1 \mathrm{H}), 6.90$ $7.04(\mathrm{~m}, 2 \mathrm{H}), 7.32-7.38(\mathrm{~m}, 2 \mathrm{H}) .{ }^{13} \mathrm{C}$ NMR $(50 \mathrm{MHz}$, $\left.\mathrm{CDCl}_{3}\right): \delta=55.4,70.5,74.7,110.5,120.9,124.9,126.1$, 130.3, 154.9, 156.1. Anal. Calcd (\%) for $\mathrm{C}_{10} \mathrm{H}_{10} \mathrm{O}_{4}: \mathrm{C}, 61.85$; H, 5.19. Found: C, 61.79; H, 5.14.

4-(3,4,5-Trimethoxyphenyl)-1,3-dioxolan-2-one (4j) 
Yield 90\% (686 mg, 2.7 mmol); brown solid; mp 133$134{ }^{\circ} \mathrm{C}$. IR $\left(\mathrm{CHCl}_{3}\right): v_{\max }=1068,1125,1243,1510,1796$ $\mathrm{cm}^{-1} .{ }^{1} \mathrm{H}$ NMR $\left(200 \mathrm{MHz}, \mathrm{CDCl}_{3}\right): \delta=3.84(\mathrm{~s}, 3 \mathrm{H}), 3.88(\mathrm{~s}$, $6 \mathrm{H}), 4.31(\mathrm{t}, J=8.3 \mathrm{~Hz}, 1 \mathrm{H}), 4.78(\mathrm{t}, J=8.4 \mathrm{~Hz}, 1 \mathrm{H}), 5.60$ $(\mathrm{t}, J=7.9 \mathrm{~Hz}, 1 \mathrm{H}), 6.54(\mathrm{~s}, 2 \mathrm{H}) .{ }^{13} \mathrm{C} \mathrm{NMR}(50 \mathrm{MHz}$, $\left.\mathrm{CDCl}_{3}\right): \delta=56.1,60.7,71.1,78.0,102.6,131.2,138.8$, 153.8, 154.5. HRMS $\left(\right.$ ESI $\left.^{+}\right): \mathrm{m} / z$ calcd for $\left(\mathrm{C}_{12} \mathrm{H}_{14} \mathrm{O}_{6}\right)^{+}[\mathrm{M}+$ $\mathrm{H}]^{+}:$255.0869; found: 255.0855. Anal. Calcd (\%) for $\mathrm{C}_{12} \mathrm{H}_{14} \mathrm{O}_{6}$ : C, 56.69; H, 5.55. Found: C, 56.62; H, 5.50. 4-(4-Fluorophenyl)-1,3-dioxolan-2-one (4k)

Yield 90\% (492 mg, $2.7 \mathrm{mmol}$ ); colorless solid; mp 91$92{ }^{\circ} \mathrm{C} . \mathrm{IR}\left(\mathrm{CHCl}_{3}\right): v_{\max }=773,840,1069,1161,1210,1385$, $1514,1818 \mathrm{~cm}^{-1} .{ }^{1} \mathrm{H}$ NMR $\left(200 \mathrm{MHz}, \mathrm{CDCl}_{3}\right): \delta=4.31(\mathrm{t}$, $J=8.2 \mathrm{~Hz}, 1 \mathrm{H}), 4.80$ (t, $J=8.3 \mathrm{~Hz}, 1 \mathrm{H}), 5.66$ (t, $J=8.0 \mathrm{~Hz}$, $1 \mathrm{H}), 7.08-7.19$ (m, $2 \mathrm{H}), 7.32-7.40(\mathrm{~m}, 2 \mathrm{H}) .{ }^{13} \mathrm{C}$ NMR (50 $\left.\mathrm{MHz}, \mathrm{CDCl}_{3}\right): \delta=71.0,77.3,116,116.5,127.9,128.0$, 131.6, 154.4, 160.84, 165.8. Anal. Calcd (\%) for $\mathrm{C}_{9} \mathrm{H}_{7} \mathrm{FO}_{3}$ : C, 59.35; H, 3.87. Found: C, 59.37; H, 3.86.

4-(4-Chlorophenyl)-1,3-dioxolan-2-one (4I)

Yield 87\% (518 mg, $2.61 \mathrm{mmol}$ ); colorless solid; mp 70-71 ${ }^{\circ} \mathrm{C}$ (lit. mp 68-69 $\left.{ }^{\circ} \mathrm{C}\right)$. IR $\left(\mathrm{CHCl}_{3}\right): v_{\max }=770,829,1071$, 1167, 1384, 1494, $1816 \mathrm{~cm}^{-1}$. ${ }^{1} \mathrm{H}$ NMR (200 MHz, $\left.\mathrm{CDCl}_{3}\right)$ : $\delta=4.29(\mathrm{t}, J=8.4 \mathrm{~Hz}, 1 \mathrm{H}), 4.80(\mathrm{t}, J=8.4 \mathrm{~Hz}, 1 \mathrm{H}), 5.65$ $(\mathrm{t}, J=7.8 \mathrm{~Hz}, 1 \mathrm{H}), 7.28-7.33(\mathrm{~m}, 2 \mathrm{H}), 7.36-7.44(\mathrm{~m}, 2 \mathrm{H})$. ${ }^{13} \mathrm{C}$ NMR (50 MHz, $\left.\mathrm{CDCl}_{3}\right): \delta=70.9,77.1,127.2,129.4$, 134.4, 135.7, 154.3. Anal. Calcd (\%) for $\mathrm{C}_{9} \mathrm{H}_{7} \mathrm{ClO}_{3}: \mathrm{C}$, 54.43; H, 3.55. Found: C, 54.37; H, 3.56. 4-(2-Bromophenyl)-1,3-dioxolan-2-one (4m)

Yield 96\% (700 mg, $2.88 \mathrm{mmol})$; gum. IR $\left(\mathrm{CHCl}_{3}\right): v_{\max }=$ $763,969,1072,1125,1159,1208,1473,1817 \mathrm{~cm}^{-1} \cdot{ }^{1} \mathrm{H}$ $\operatorname{NMR}\left(200 \mathrm{MHz}, \mathrm{CDCl}_{3}\right): \delta=4.24(\mathrm{dd}, J=6.8,1.7 \mathrm{~Hz}, 1 \mathrm{H})$, $4.99(\mathrm{t}, J=8.4 \mathrm{~Hz}, 1 \mathrm{H}), 5.94(\mathrm{t}, J=8.0 \mathrm{~Hz}, 1 \mathrm{H}), 7.28-7.39$ (m, 1 H), 7.43-7.62 (m, 3 H). ${ }^{13} \mathrm{C}$ NMR (50 MHz, $\left.\mathrm{CDCl}_{3}\right)$ : $\delta=70.4,76.3,120.1,126.0,128.1,130.4,132.96,136.3$, 154.3. Anal. Calcd (\%) for $\mathrm{C}_{9} \mathrm{H}_{7} \mathrm{BrO}_{3}: \mathrm{C}, 44.47 ; \mathrm{H}, 2.90$. Found: C, 44.45; H, 2.86.

4-[4-(Trifluoromethyl)phenyl]-1,3-dioxolan-2-one (4n) Yield 98\% (683 mg, $2.94 \mathrm{mmol})$; gum. IR $\left(\mathrm{CHCl}_{3}\right): v_{\max }=$ $771,844,1071,1167,1264,1327,1426,1822 \mathrm{~cm}^{-1} .{ }^{1} \mathrm{H}$ $\operatorname{NMR}\left(200 \mathrm{MHz}, \mathrm{CDCl}_{3}\right): \delta=4.30(\mathrm{t}, J=7.8 \mathrm{~Hz}, 1 \mathrm{H}), 4.85$ $(\mathrm{t}, J=8.4 \mathrm{~Hz}, 1 \mathrm{H}), 5.74(\mathrm{t}, J=7.9 \mathrm{~Hz}, 1 \mathrm{H}), 7.48(\mathrm{~d}, J=8.0$ $\mathrm{Hz}, 1 \mathrm{H}), 7.70-7.74(\mathrm{~d}, J=8.2 \mathrm{~Hz}, 2 \mathrm{H}) .{ }^{13} \mathrm{C} \mathrm{NMR}(50 \mathrm{MHz}$, $\left.\mathrm{CDCl}_{3}\right): \delta=70.8,76.8,126.0,126.2,126.3,126.4,139.9$, 154.2. HRMS $\left(\mathrm{ESI}^{+}\right): \mathrm{m} / z$ calcd for $\left(\mathrm{C}_{10} \mathrm{H}_{7} \mathrm{~F}_{3} \mathrm{O}_{3}\right)^{+}[\mathrm{M}+\mathrm{H}]^{+}$: 233.0426; found: 233.0426. Anal. Calcd (\%) for $\mathrm{C}_{10} \mathrm{H}_{7} \mathrm{~F}_{3} \mathrm{O}_{3}$ : C, 51.74; H, 3.04. Found: C, 51.71; H, 3.06.

4-(2-Nitrophenyl)-1,3-dioxolan-2-one (4o)

Yield 79\% (496 mg, $2.37 \mathrm{mmol}$ ); brown solid; $\mathrm{mp} 95-96^{\circ} \mathrm{C}$. IR $\left(\mathrm{CHCl}_{3}\right): v_{\max }=1073,1167,1350,1527,1819 \mathrm{~cm}^{-1} .{ }^{1} \mathrm{H}$ $\operatorname{NMR}\left(200 \mathrm{MHz}, \mathrm{CDCl}_{3}\right): \delta=4.27(\mathrm{dd}, J=9.0,3.3 \mathrm{~Hz}, 1 \mathrm{H})$, $5.17(\mathrm{t}, J=8.9 \mathrm{~Hz}, 1 \mathrm{H}), 6.28(\mathrm{dd}, J=8.7,2.7 \mathrm{~Hz}, 1 \mathrm{H})$,

$7.60-7.68(\mathrm{~m}, 1 \mathrm{H}), 7.83(\mathrm{~d}, J=4.0 \mathrm{~Hz}, 2 \mathrm{H}), 8.26(\mathrm{~d}, J=8.2$ $\mathrm{Hz}, 1 \mathrm{H}) .{ }^{13} \mathrm{C} \mathrm{NMR}\left(50 \mathrm{MHz}, \mathrm{CDCl}_{3}\right): \delta=71.2,74.3,125.6$, 126.2, 129.9, 134.1, 135.1, 145.9, 154.2 Anal. Calcd (\%) for $\mathrm{C}_{9} \mathrm{H}_{7} \mathrm{NO}_{5}: \mathrm{C}, 51.68 ; \mathrm{H}, 3.37 ; \mathrm{N}, 6.70$. Found: C, 51.71; H, $3.35 ; \mathrm{N}, 6.66$.

4-(3-Nitrophenyl)-1,3-dioxolan-2-one (4p)

Yield 75\% (470 mg, $2.25 \mathrm{mmol}$ ); brown solid; $\mathrm{mp} 97-98^{\circ} \mathrm{C}$. IR $\left(\mathrm{CHCl}_{3}\right): v_{\max }=1071,1166,1349,1530,1805 \mathrm{~cm}^{-1} .{ }^{1} \mathrm{H}$ $\operatorname{NMR}\left(200 \mathrm{MHz}, \mathrm{CDCl}_{3}\right): \delta=4.35(\mathrm{dd}, J=8.7,1.1 \mathrm{~Hz}, 1 \mathrm{H})$, $4.91(\mathrm{t}, J=8.5 \mathrm{~Hz}, 1 \mathrm{H}), 5.81(\mathrm{t}, J=7.8 \mathrm{~Hz}, 1 \mathrm{H}), 7.64-7.78$ $(\mathrm{m}, 2 \mathrm{H}), 8.24-8.33(\mathrm{~m}, 2 \mathrm{H}) .{ }^{13} \mathrm{C} \mathrm{NMR}\left(50 \mathrm{MHz}, \mathrm{CDCl}_{3}\right): \delta$ $=70.7,76.3,120.9,124.5,130.5,131.4,138.2,148.6,153.8$. HRMS (ESI $\left.{ }^{+}\right): m / z$ calcd for $\left(\mathrm{C}_{9} \mathrm{H}_{7} \mathrm{NO}_{5}\right)^{+}[\mathrm{M}+\mathrm{Na}]^{+}$: 232.0221; found: 232.0214 . Anal. Calcd (\%) for $\mathrm{C}_{9} \mathrm{H}_{7} \mathrm{NO}_{5}$ : C, 51.68; H, 3.37; N, 6.70. Found: C, 51.72; H, 3.34; N, 6.68.

\section{4-\{Benzo[d][1,3]dioxol-5-yl\}-1,3-dioxolan-2-one (4q)}

Yield 86\% (537 mg, $2.58 \mathrm{mmol})$; gum. IR $\left(\mathrm{CHCl}_{3}\right): v_{\max }=$ $1070,1164,1251,1505,1791 \mathrm{~cm}^{-1} .{ }^{1} \mathrm{H}$ NMR $(200 \mathrm{MHz}$, $\left.\mathrm{CDCl}_{3}\right): \delta=4.32(\mathrm{t}, J=8.4 \mathrm{~Hz}, 1 \mathrm{H}), 4.75(\mathrm{t}, J=8.5 \mathrm{~Hz}, 1$ $\mathrm{H}), 5.58(\mathrm{t}, J=8.0 \mathrm{~Hz}, 1 \mathrm{H}), 6.01(\mathrm{~s}, 2 \mathrm{H}), 6.84(\mathrm{~s}, 3 \mathrm{H}) .{ }^{13} \mathrm{C}$ $\operatorname{NMR}\left(50 \mathrm{MHz}, \mathrm{CDCl}_{3}\right): \delta=71.0,78.0,101.5,106.1,108.6$, 120.3, 129.2, 148.5, 148.8, 154.6. Anal. Calcd (\%) for $\mathrm{C}_{10} \mathrm{H}_{8} \mathrm{O}_{5}: \mathrm{C}, 57.70 ; \mathrm{H}, 3.87$. Found: $\mathrm{C}, 57.72 ; \mathrm{H}, 3.84$. 4-[2-(Benzyloxy)ethyl]-1,3-dioxolan-2-one (4r) Yield $80 \%$ (533 mg, $2.4 \mathrm{mmol})$; gum. IR $\left(\mathrm{CHCl}_{3}\right): v_{\max }=$ 1061, 1173, 1364, 1454, $1794 \mathrm{~cm}^{-1} .{ }^{1} \mathrm{H}$ NMR (200 MHz, $\left.\mathrm{CDCl}_{3}\right): \delta=2.02-2.10(\mathrm{~m}, 2 \mathrm{H}), 3.58-3.65(\mathrm{~m}, 2 \mathrm{H}), 4.17$ $(\mathrm{dd}, J=8.4,1.0 \mathrm{~Hz}, 1 \mathrm{H}), 4.47-4.52(\mathrm{~m}, 3 \mathrm{H}), 4.79-4.93(\mathrm{~m}$, $1 \mathrm{H}), 7.29-7.40(\mathrm{~m}, 5 \mathrm{H}) \cdot{ }^{13} \mathrm{C} \mathrm{NMR}\left(50 \mathrm{MHz}, \mathrm{CDCl}_{3}\right): \delta=$ $32.0,33.9,61.5,65.3,69.1,69.6,73.1,73.3,75.0,127.5$, 127.8, 128.3, 128.4, 137.5, 154.7. Anal. Calcd (\%) for $\mathrm{C}_{12} \mathrm{H}_{14} \mathrm{O}_{4}$ : C, 64.85; H, 6.35. Found: C, 64.82; H, 6.31. 4-Phenethyl-1,3-dioxolan-2-one (4s)

Yield 77\% (444 mg, $2.31 \mathrm{mmol})$; gum. IR $\left(\mathrm{CHCl}_{3}\right): v_{\max }=$ $1061,1165,1796 \mathrm{~cm}^{-1} .{ }^{1} \mathrm{H}$ NMR $\left(200 \mathrm{MHz}, \mathrm{CDCl}_{3}\right): \delta=$ $1.94-2.17$ (m, 2 H), 2.72-2.91 (m, $2 \mathrm{H}), 4.01$ (dd, $J=8.3$, $1.1 \mathrm{~Hz}, 1 \mathrm{H}), 4.44(\mathrm{t}, J=8.2 \mathrm{~Hz}, 1 \mathrm{H}), 4.62-4.68(\mathrm{~m}, 1 \mathrm{H})$, 7.15-7.34 (m, $5 \mathrm{H}) .{ }^{13} \mathrm{C}$ NMR (50 MHz, $\left.\mathrm{CDCl}_{3}\right): \delta=30.8$, 35.6, 69.1, 75.8, 126.5, 128.3, 128.7, 139.6, 154.6. Anal. Calcd (\%) for $\mathrm{C}_{11} \mathrm{H}_{12} \mathrm{O}_{3}: \mathrm{C}, 68.74 ; \mathrm{H}, 6.29$. Found: $\mathrm{C}, 68.71$; H, 6.26 .

4,5-Dimethoxy-2-(2-oxo-1,3-dioxolan-4-yl)benzonitrile (4t)

Yield 83\% $(690.42,2.49 \mathrm{mmol})$; gum. IR $\left(\mathrm{CHCl}_{3}\right): v_{\max }=$ 1064, 1168, 1270, 1516, 1793, $2218 \mathrm{~cm}^{-1} .{ }^{1} \mathrm{H}$ NMR $(200$ $\left.\mathrm{MHz}, \mathrm{CDCl}_{3}\right): \delta=2.09(\mathrm{q}, J=7.8 \mathrm{~Hz}, 1 \mathrm{H}), 2.89-2.98(\mathrm{~m}$, $1 \mathrm{H}), 3.89$ (s, $3 \mathrm{H}), 3.94$ (s, $3 \mathrm{H}), 4.14$ (dd, $J=8.3,1.6 \mathrm{~Hz}$, $1 \mathrm{H}), 4.54(\mathrm{t}, J=8.2 \mathrm{~Hz}, 1 \mathrm{H}), 4.62-4.73(\mathrm{~m}, 1 \mathrm{H}), 6.80(\mathrm{~s}$, $1 \mathrm{H}), 7.03(\mathrm{~s}, 1 \mathrm{H}) .{ }^{13} \mathrm{C}$ NMR $\left(50 \mathrm{MHz}, \mathrm{CDCl}_{3}\right): \delta=29.5$, 35.0, 56.1, 69.0, 75.6, 103.0, 112.3, 114.2, 118.0, 138.1,

147.9, 152.9, 154.5. HRMS $\left(\mathrm{ESI}^{+}\right): \mathrm{m} / \mathrm{z}$ calcd for $\left(\mathrm{C}_{14} \mathrm{H}_{15} \mathrm{NO}_{5}\right)^{+}[\mathrm{M}+\mathrm{H}]^{+}: 278.1023$; found: 278.1026 . Anal. Calcd (\%) for $\mathrm{C}_{14} \mathrm{H}_{15} \mathrm{NO}_{5} \mathrm{C}, 60.64 ; \mathrm{H}, 5.45 ; \mathrm{N}, 5.05$. Found: C, $60.60 ; \mathrm{H}, 5.40 ; \mathrm{N}, 5.10$.

\section{tert-Butyl \{(S)-1-[(S)-2-Oxo-1,3-dioxolan-4-yl]-2-} phenylethyl $\}$ carbamate $(4 \mathrm{u})$

Yield 79\% (728 mg, $2.37 \mathrm{mmol}$ ); colorless solid; mp 148 $149^{\circ} \mathrm{C}$. IR $\left(\mathrm{CHCl}_{3}\right): v_{\max }=1061,1169,1249,1366,1689$, $1800 \mathrm{~cm}^{-1}$. ${ }^{1} \mathrm{H}$ NMR $\left(200 \mathrm{MHz}, \mathrm{CDCl}_{3}\right): \delta=1.40(\mathrm{~s}, 9 \mathrm{H})$, $2.82-2.89(\mathrm{~m}, 2 \mathrm{H}), 4.09(\mathrm{~m}, 1 \mathrm{H}), 4.28-4.44(\mathrm{~m}, 2 \mathrm{H}), 4.67$ (m, $2 \mathrm{H}), 7.22-7.33(\mathrm{~m}, 5 \mathrm{H}) .{ }^{13} \mathrm{C} \mathrm{NMR}\left(50 \mathrm{MHz}, \mathrm{CDCl}_{3}\right): \delta$ $=28.2,38.5,52.8,66.5,76.1,80.4,127.1,128.88,129.2$, 136.4, 154.6, 155.9. Anal. Calcd (\%) for $\mathrm{C}_{16} \mathrm{H}_{21} \mathrm{NO}_{5}$ : C, $62.53 ; \mathrm{H}, 6.89 ; \mathrm{N}, 4.56$. Found: C, 62.57; H, 6.91; N, 4.60 . 4-[4-(Methylthio)phenyl]-1,3-dioxolan-2-one (4v) Yield $90 \%$ (573 mg, $2.73 \mathrm{mmol})$; yellow solid; mp 55$57^{\circ} \mathrm{C} . \mathrm{IR}\left(\mathrm{CHCl}_{3}\right): v_{\max }=817,895,1062,1173,1384,1514$, $1767 \mathrm{~cm}^{-1} .{ }^{1} \mathrm{H}$ NMR $\left(200 \mathrm{MHz}, \mathrm{CDCl}_{3}\right): \delta=2.49(\mathrm{~s}, 3 \mathrm{H})$, $4.30(\mathrm{t}, J=8.1 \mathrm{~Hz}, 1 \mathrm{H}), 4.76(\mathrm{t}, J=8.2 \mathrm{~Hz}, 1 \mathrm{H}), 5.61(\mathrm{t}$, $J=8.0 \mathrm{~Hz}, 1 \mathrm{H}), 7.27(\mathrm{~s}, 4 \mathrm{H}) .{ }^{13} \mathrm{C} \mathrm{NMR}\left(100 \mathrm{MHz}, \mathrm{CDCl}_{3}\right)$ : $\delta=15.0,70.7,77.5,125.8,131.9,140.7,154.5$. Anal. Calcd (\%) for $\mathrm{C}_{10} \mathrm{H}_{10} \mathrm{SO}_{3}: \mathrm{C}, 57.13 ; \mathrm{H}, 4.79$. Found: $\mathrm{C}, 57.17 ; \mathrm{H}$, 4.86. HRMS (ESI $\left.{ }^{+}\right): m / z$ calcd for $\left(\mathrm{C}_{10} \mathrm{H}_{10} \mathrm{O}_{3} \mathrm{~S}\right)^{+}[\mathrm{M}+\mathrm{H}]^{+}$: 211.0429; found: 211.0441

2-Iodo-1-phenylethan-1-ol (3a)

Gum. IR $\left(\mathrm{CHCl}_{3}\right): v_{\max }=699,1054,1174,1452,3404 \mathrm{~cm}^{-1}$. ${ }^{1} \mathrm{H} \mathrm{NMR}\left(200 \mathrm{MHz}, \mathrm{CDCl}_{3}\right): \delta=2.44($ br s, $1 \mathrm{H}), 3.33-3.52$ (m, $2 \mathrm{H}), 4.80-4.84(\mathrm{~m}, 1 \mathrm{H}), 7.31-7.38(\mathrm{~m}, 5 \mathrm{H}) .{ }^{13} \mathrm{C} \mathrm{NMR}$ $\left(50 \mathrm{MHz}, \mathrm{CDCl}_{3}\right): \delta=1502,74.0,125.7,128.3,128.6$, 141.1. Anal. Calcd (\%) for $\mathrm{C}_{8} \mathrm{H}_{9} \mathrm{IO}: \mathrm{C}, 38.74 ; \mathrm{H}, 3.66$. Found: C, 38.70; H, 3.63. 